\title{
Bound on the Ionization Energy of Large Atoms
}

L. A. Seco ${ }^{1 \star}$, I. M. Sigal ${ }^{2 \star \star \star \star \star}$ and J. P. Solovej ${ }^{1 \star \star \star \star}$

${ }^{1}$ Department of Mathematics, Princeton University, Princeton, NJ 08544, USA

${ }^{2}$ Department of Mathematics, University of Toronto, Toronto, Canada M5S1A1

\begin{abstract}
We present a simple argument which gives a bound on the ionization energy of large atoms that implies the bound on the excess charge of Fefferman and Seco [2].
\end{abstract}

\section{Introduction}

A system consisting of a nucleus of charge $Z$ and $N$ electrons is described by the Schrödinger operator

$$
H_{N, Z}=\sum_{i}^{N}\left(-\Delta_{i}-\frac{Z}{\left|x_{i}\right|}\right)+\sum_{1 \leqq i<j \leqq N} \frac{1}{\left|x_{i}-x_{j}\right|}
$$

acting on the antisymmetric space $\mathscr{H}_{F}=\bigwedge_{i=1}^{N}\left(L^{2}\left(\mathbb{R}^{3}\right) \otimes \mathbb{C}^{2}\right)$. Here we have assumed for simplicity that the nucleus is infinitely heavy. We call such a system an atom.

The ground state energy of the atom is

$$
E(N, Z)=\inf \operatorname{spec}_{\mathscr{H}} H_{N, Z}
$$

and the ionization energy is defined as

$$
I(N, Z)=E(N-1, Z)-E(N, Z) .
$$

This is the energy which binds the atom together. It is well known that there is a critical number of electrons $N_{c}(Z)$ such that

$$
I\left(N_{c}, Z\right)>0 \text { and } I(N, Z)=0 \text { if } N>N_{c}
$$

* Supported by a Sloan Dissertation Fellowship. Address from September 1989: Department of Mathematics, Caltech, Pasadena, CA 91125, USA

$\star \star$ I. W. Killam fellow

$\star \star \star$ Supported in part by NSERC Grant N. A7901

$\star \star \star \star$ Supported by a Danish Research Academy Fellowship and U.S. National Science Foundation Grant PHY-85-15288-A03. Address from September 1989: Department of Mathematics, University of Michigan, Ann Arbor, MI 48109, USA 
(see $[11,12,15,16])$. Using a variational estimate one can derive the lower bound $N_{c}(Z) \geqq Z$ ([20]). It was shown in [8] and [17] (for large $Z$ ) that $N_{c}(Z)<2 Z+1$. Define the excess charge as

$$
Q_{c}(Z)=N_{c}(Z)-Z .
$$

For $N \leqq N_{c}$ the operator $H_{N, Z}$ has a ground state $\psi_{N, Z} \in \mathscr{H}_{F}$.

We define the radius $R(N, Z)$ of the atom by

$$
\int_{|x| \leqq R(N, Z)} \rho_{N, Z} d x=N-1,
$$

where $\rho_{N, Z}$ is the one-electron density

$$
\rho_{N, Z}(x)=N \sum_{\sigma=1,2} \int\left|\psi_{N, Z}\left(x, \sigma ; x_{2}, \sigma_{2} ; \cdots ; x_{N}, \sigma_{N}\right)\right|^{2} d\left(x_{2}, \sigma_{2}\right) \cdots d\left(x_{N}, \sigma_{N}\right),
$$

$x_{i}$ are the space variables and $\sigma_{i}$ the spin variables, $\int d(x, \sigma)=\sum_{\sigma} \int d x$. (Throughout most of the paper explicit mention of the spin variables will be omitted.) Outside $R(N, Z)$ there is an average of one electron.

It is expected that as $Z \rightarrow \infty$

$$
Q_{c}(Z), I(Z, Z), R(Z, Z)=O(1) .
$$

In Thomas-Fermi theory it has been known for some time that as $Z \rightarrow \infty$ the atomic structure shows a universal behavior, which is to say that the quantities in (6) actually converge to non-zero values as $Z \rightarrow \infty$ (see [7]). In the present paper we will indeed compare with TF theory. In the Thomas-Fermi-von Weizsäcker theory universality was recently proved in [18].

It follows from $[8,17]$ that

$$
Q_{c}(Z) \leqq C Z, \quad I(Z, Z) \leqq C Z^{4 / 3} \text { and } \quad R(Z, Z) \geqq C Z^{-1 / 3} \text {. }
$$

In [9] it was proved that $Q_{c}(Z)=o(Z)$. This has recently been improved in [2] (an announcement was made in [3]) to $Q_{c}(Z) \leqq C Z^{1-\alpha}$ with $\alpha=9 / 56$.

Our main result is

Theorem 1. For $Z \leqq N \leqq N_{c}$ and with $\alpha=9 / 56$,

$$
I(N, Z) \leqq C_{1} Z^{(4 / 3)(1-\alpha)}-C_{2}(N-Z) Z^{(1 / 3)(1-\alpha)} .
$$

We get as an immediate consequence

\section{Corollary 2.}

$$
Q_{c}(Z) \leqq C Z^{1-\alpha},
$$

and for $N \geqq Z$,

$$
I(N, Z) \leqq C Z^{(4 / 3)(1-\alpha)} .
$$

As a very easy consequence of the proof of Theorem 1 we also find (see Lemma 7)

Theorem 3. For $N \geqq Z$,

$$
R(N, Z) \geqq C Z^{-(1 / 3)(1-\alpha)}
$$


We prove Theorem 1 by first proving a general estimate on $I(N, Z)$ which for an arbitrary radius $R$ bounds $I$ in terms of quantities we call the screening charge at radius $R$, the excess charge at radius $R$, and the 2-point correlation outside $R$. This general bound is given in Sect. 2. In Sect. 4 we estimate the above quantities.

Our method emphasizes the importance of controlling the 2-point correlation function

$$
\rho^{(2)}(x, y)=N(N-1) \sum_{\sigma_{1}, \sigma_{2}} \int\left|\psi\left(x, \sigma_{1} ; y, \sigma_{2} ; \cdots ; x_{N}, \sigma_{N}\right)\right|^{2} d\left(x_{3}, \sigma_{3}\right) \cdots d\left(x_{N}, \sigma_{N}\right),
$$

(we will often omit the subscripts $N, Z$ ). In fact the key step is to estimate the truncated correlation function

$$
\rho^{(2)}(x, y)-\rho(x) \rho(y),
$$

where $\rho=\rho_{N, Z}$, this is done in Sect. 3 Lemma 5.

Now we explain the origin of the number $\alpha$ in (8). Define the effective particle (or quasiparticle in physicists' terminology) Hamiltonian

$$
H_{N, Z}^{\mathrm{ind}}=\sum_{i=1}^{N}\left(-\Delta_{i}-\phi\left(x_{i}\right)\right)-D_{\mathrm{TF}}
$$

acting on $\mathscr{H}_{F}$. Here $\phi$ is the smeared Thomas-Fermi potential

$$
\phi(x)=\frac{Z}{|x|}-\frac{1}{|x|} * \rho_{\mathrm{TF}}(x),{ }^{*} \varphi,
$$

with a $C_{0}^{\infty}$ cut-off function $\varphi$ introduced in (12) below, where $\rho_{\mathrm{TF}}$ is the Thomas-Fermi density for a neutral atom with nuclear charge $Z$ (see [7] for a review of Thomas-Fermi theory), and

$$
D_{\mathrm{TF}}=\iint \frac{\rho_{\mathrm{TF}}(x) \rho_{\mathrm{TF}}(y)}{|x-y|} .
$$

It is a fundamental result of Lieb and Simon ([10], see also [7] and [19] for a proof) that there exists $0<b$ such that for $N \geqq Z$ - const,

$$
H_{N, Z}^{\text {ind }} \geqq E(N, Z)-C Z^{7 / 3-b}
$$

for some constant $C$. Finding the optimal $b$ is a hard problem requiring an understanding of the ground state structure. Presently the best known result is $b \geqq 3 / 8([4,13,14]$ the previous result in [7], was $b \geqq 1 / 30)$. This estimate involves the proof of the Scott conjecture. It is believed that the optimal value for $b$ is $2 / 3$. Our arguments hold as long as $b \leqq 2 / 3$.

Note that in the result of [2] as well as in our result $\alpha=3 b / 7$.

\section{General Argument}

Given $\delta$, choose $\theta_{1} \in C^{\infty}\left(\mathbb{R}_{+}\right)$with $0 \leqq \theta_{0} \leqq 1$, and $\theta_{1}(t)=0$ if $t \leqq 1-\delta, \theta_{1}(t)=1$ if $t \geqq 1$.

For all $R$, let $\theta_{R}$ and $\lambda_{R}: \mathbb{R}^{3} \mapsto \mathbb{R}^{3}$ be given by

$$
\theta_{R}(x)=\theta_{1}(|x| / R)^{2} \quad \text { and } \quad \lambda_{R}(x)=\left(1-\theta_{R}(x)\right) \text {. }
$$


We define ( $\delta$ is fixed):

the excess charge at radius $R$,

$$
Q(R)=\int \rho(x) \theta_{R}(x) d x,
$$

the screening charge at radius $R$,

$$
v(R)=Z-\frac{1}{Q(R)} \int \rho^{(2)}(x, y) \frac{|x|}{|x-y|} \theta_{R}(x) \lambda_{R}(y) d x d y,
$$

the normalized 2-point correlation outside $R$,

$$
K(R)=\frac{1}{Q(R)} \int \rho^{(2)}(x, y) \theta_{R}(x) \theta_{R}(y) d x d y .
$$

We will prove an upper bound to the ionization energy in terms of these quantities by using a very simple trick which goes back to Benguria (see [7]) and was used in [8] to prove $N_{c}<2 Z+1$. The idea here is to use the trick on the outside problem $(|x|>R)$. The same method was used in [18]. Below, $\delta>0$ is fixed and the dependence on $\delta$ of quantities of interest and constants is not displayed.

Theorem 4. For all $\delta>0$ and $R>0$,

$$
I \leqq\left[v(R)-\frac{1}{2} K(R)\right] R^{-1}+X(R),
$$

where the error term is bounded by

$$
X \leqq c R^{-2} \frac{Q(R(1-\delta))}{Q(R)} .
$$

Proof. From the IMS formula (see e.g. [1]) we find

$$
\begin{aligned}
E_{N, Z} \int \rho(x)|x| \theta_{R}(x) d x= & \sum_{i}\left\langle\psi_{N, Z}\left|\theta_{R}\left(x_{i}\right)\right| x_{i}\left|H_{N, Z}\right| \psi_{N, Z}\right\rangle \\
= & \sum_{i}\left\langle\left.\psi_{N, Z}\left|\theta_{R}\left(x_{i}\right)^{1 / 2}\right| x_{i}\right|^{1 / 2} H_{N, Z} \theta_{R}\left(x_{i}\right)^{1 / 2}\left|x_{i}\right|^{1 / 2} \mid \psi_{N, Z}\right\rangle \\
& -\sum_{i}\left\langle\left.\psi_{N, Z}|| \nabla\left(\theta_{R}\left(x_{i}\right)^{1 / 2}\left|x_{i}\right|^{1 / 2}\right)\right|^{2} \mid \psi_{N, Z}\right\rangle .
\end{aligned}
$$

Isolating the contribution of the $i^{\text {th }}$ electron in the $i^{\text {th }}$ term in the first sum on the right-hand side, we obtain

$$
\begin{aligned}
E_{N, Z} \int \rho(x)|x| \theta_{R}(x) d x \geqq & E_{N-1, Z} \int \rho(x)|x| \theta_{R}(x) d x \\
& +\sum_{i}\left\langle\psi_{N, Z}\left|\theta_{R}\left(x_{i}\right)\right| x_{i}\left|\left(-\frac{Z}{\left|x_{i}\right|}+\sum_{j, j \neq i} \frac{1}{\left|x_{j}-x_{i}\right|}\right)\right| \psi_{N, Z}\right\rangle \\
& -\sum_{i} \int\left|\nabla\left(\theta_{R}\left(x_{i}\right)^{1 / 2}\left|x_{i}\right|^{1 / 2}\right)\right|^{2}|\psi|^{2} d x .
\end{aligned}
$$

Using $\left|\nabla\left(\theta_{R}(x)^{1 / 2}|x|^{1 / 2}\right)\right| \leqq c R^{-1} \theta_{(1-\delta) R}(x)$ and $\int \rho(x)|x| \theta_{R}(x) d x \geqq R Q(R)$, we rewrite this inequality as

$$
-R I Q(R) \geqq-Z Q(R)+\int \rho^{(2)}(x, y) \frac{|x|}{|x-y|} \theta_{R}(x) d x d y-c Q(R(1-\delta)) R^{-1} .
$$

In [8] the error term (the last term above) could be ignored by use of the uncertainty 
principle: $\int(\nabla u)^{2} \geqq(1 / 4) \int u^{2} /|x|^{2}$. Here the uncertainty principle can be used to improve $c$, but this is not necessary.

The trick is now to symmetrize and use the triangle inequality

$$
\begin{aligned}
R I Q(R) & \leqq v(R) Q(R)-\int \rho^{(2)}(x, y) \frac{|x|}{|x-y|} \theta_{R}(x) \theta_{R}(y) d x d y+c Q(R(1-\delta)) R^{-1} \\
& =v(R) Q(R)-\frac{1}{2} \int \rho^{(2)}(x, y) \frac{|x|+|y|}{|x-y|} \theta_{R}(x) \theta_{R}(y) d x d y+c Q(R(1-\delta)) R^{-1} \\
& \leqq v(R) Q(R)-\frac{1}{2} K(R) Q(R)+c Q(R(1-\delta)) R^{-1} .
\end{aligned}
$$

\section{Estimates on the Density and Correlation Function}

The idea of comparing the exact charge distribution with the Thomas-Fermi one goes back to [10] with an effective estimate derived in [2]. We extend this idea and, in particular, the method of [2] further to estimates of the 2-point correlation. Choose $\varphi_{1} \in C_{0}^{\infty}\left(\mathbb{R}^{3}\right)$ radially symmetric, positive and with $\int \varphi_{1}=1$. Let

$$
\varphi(x)=\varphi_{Z}(x)=Z^{2} \varphi_{1}\left(Z^{2 / 3} x\right),
$$

then $\int \varphi=1$. With $\rho_{\mathrm{TF}}$ the Thomas-Fermi density for a neutral atom with nuclear charge $Z$, we define a function $K_{N}: \mathbb{R}^{3 N} \rightarrow \mathbb{R}_{+}$by

$$
K_{N}\left(x_{1}, \ldots, x_{N}\right)=D\left(\sum_{i=1}^{N} \varphi\left(\cdot-x_{i}\right)-\rho_{\mathrm{TF}}, \sum_{i=1}^{N} \varphi\left(\cdot-x_{i}\right)-\rho_{\mathrm{TF}}\right),
$$

where

$$
D(f, g)=\frac{1}{2} \iint f(x)|x-y|^{-1} g(x) d x d y .
$$

We derive the key inequality from [2], i.e., (15) below, which also plays an essential role in our analysis. Main steps in this derivation go back to [6]. The first step is to smear the point charges. Namely using Newton's screening Theorem, one obtains

$$
\sum_{1 \leqq i<j \leqq N}\left|x_{i}-x_{j}\right|^{-1} \geqq D\left(\rho_{\underline{x}}, \rho_{\underline{x}}\right)-c Z^{5 / 3}
$$

where

$$
\rho_{\underline{x}}=\sum_{i=1}^{N} \varphi\left(x-x_{i}\right), \quad \underline{x}=\left(x_{1}, \ldots, x_{N}\right),
$$

is the random variable for the smeared charge density and the last term in (14) comes from the self-energy, $D(\varphi, \varphi)$ of the smeared charges.

The next idea is that in the ground state $\rho_{\underline{x}}$ must look essentially as $\rho_{\mathrm{TF}}$. With this in mind we write

$$
D\left(\rho_{\underline{x}}, \rho_{\underline{x}}\right)=\int \rho_{\underline{x}}\left(|x|^{-1} * \rho_{\mathrm{TF}}\right) d x+K_{N}(\underline{x})-2 D\left(\rho_{\mathrm{TF}}, \rho_{\mathrm{TF}}\right) .
$$

The last two relations lead to the representation

$$
H_{N, Z} \geqq H_{N, Z}^{\text {ind }}+K_{N}(\underline{x})+O\left(Z^{5 / 3}\right) .
$$


Combining this with (10), we arrive at the desired operator estimate

$$
H_{N, Z} \geqq E(N, Z)+K_{N}\left(x_{1}, \ldots, x_{N}\right)-C_{0} Z^{7 / 3-b} .
$$

As with (10) this estimate is proven only for $N$ with $Z$ - const $\leqq N$.

Our main estimate is

Lemma 5. Given $\sqrt{\theta} \in C^{\infty}\left(\mathbb{R}^{3}\right)$ with $0 \leqq \theta \leqq 1, \operatorname{supp} \theta \subset\{|x| \geqq R\}$ and $|\nabla \sqrt{\theta}|<c_{1} R^{-1}$ and $\chi \in C^{\infty}\left(\mathbb{R}^{3} \times \mathbb{R}^{3}\right)$ with $0 \leqq \chi$ and $\chi_{x}=\chi(x, \cdot)$ compactly supported. Then for all $N$ with $Z \leqq N \leqq N_{c}(Z)$,

$$
\begin{aligned}
& \left|\int\left[\rho^{(2)}(x, y)-\rho_{\mathrm{TF}}(y) \rho(x)\right] \theta(x) \chi(x, y) d x d y\right| \\
& \leqq C \sup _{x}\left\|\nabla_{y} \chi_{x}\right\|_{L^{2}\left(\mathbb{R}^{3}\right)}\left\{\left(Z^{(7 / 3-b)}+Z R^{-1}\right) \int \rho(x) \theta(x) d x+Z R^{-2}\right\}^{1 / 2} \\
& \quad \cdot\left\{\int \rho(x) \theta(x) d x\right\}^{1 / 2}+C Z^{1 / 3}\left\|\nabla_{y} \chi\right\|_{L^{\infty}} \int \rho(x) \theta(x) d x,
\end{aligned}
$$

where $\rho$ and $\rho^{(2)}$ are the ground state density and correlation function. $C$ depends only on $C_{0}$ and $\varphi_{1}$.

Remark. The reason for the rather peculiar cutoff in (16) will be clear in Lemma 8 below.

Proof. Define the particle number random variables $N_{x}: \mathbb{R}^{N-1} \rightarrow \mathbb{R}_{+}$and $N_{x}^{\mathrm{TF}} \in \mathbb{R}_{+}$ by

$$
N_{x}\left(x_{2}, \ldots, x_{N}\right)=\sum_{i=2}^{N} \chi_{x} * \varphi\left(x_{i}\right) \quad \text { and } \quad N_{x}^{\mathrm{TF}}=\int \rho_{\mathrm{TF}}(y) \chi_{x}(y) d y
$$

Then

$$
\begin{aligned}
& \left|\int\left[\rho^{(2)}(x, y)-\rho_{\mathrm{TF}}(y) \rho(x)\right] \theta(x) \chi(x, y) d x d y\right| \\
& \leqq \int \rho^{(2)}(x, y)\left|\chi_{x} * \varphi(y)-\chi_{x}(y)\right| \theta(x) d x d y \\
& +\left.N\left|\int\right| \psi\left(x, x_{2}, \ldots, x_{N}\right)\right|^{2}\left(N_{x}\left(x_{2}, \ldots, x_{N}\right)-N_{x}^{\mathrm{TF}}\right) d x_{2} \cdots d x_{N} \theta(x) d x \mid \\
& \leqq C_{2} Z^{1 / 3}\left\|\nabla_{y} \chi\right\|_{L^{\infty}} \int \rho(x) \theta(x) d x \\
& +\left\{\left.N\left|\int\right| \psi\left(x, x_{2}, \ldots, x_{N}\right)\right|^{2}\left|N_{x}-N_{x}^{\mathrm{TF}}\right|^{2} \theta(x) d x d x_{2} \cdots d x_{N}\right\}^{1 / 2} \\
& \cdot\left\{\int \rho(x) \theta(x) d x\right\}^{1 / 2},
\end{aligned}
$$

where we have used Cauchy-Schwarz inequality.

Since

$$
N_{x}\left(x_{2}, \ldots, x_{N}\right)-N_{x}^{\mathrm{TF}}=\int\left(\sum_{i=2}^{N} \varphi\left(y-x_{i}\right)-\rho_{\mathrm{TF}}(y)\right) \chi(x, y) d y,
$$

we get again from Cauchy-Schwarz

$$
\begin{aligned}
\left|N_{x}-N_{x}^{\mathrm{TF}}\right|^{2} & \leqq\left.\int\left|\hat{\chi}_{x}(\xi)\right|^{2}|\xi|^{2} d \xi \int\left|\left(\sum_{i=2}^{N} \varphi\left(y-x_{i}\right)-\rho_{\mathrm{TF}}(y)\right) \hat{(\xi)}\right|\right|^{2}|\xi|^{-2} d \xi \\
& \leqq C\left\|\nabla \chi_{x}\right\|_{L^{2}\left(\mathbb{R}^{3}\right)}^{2} K_{N-1}\left(x_{2}, \ldots, x_{N}\right),
\end{aligned}
$$

where ${ }^{\wedge}$ denotes Fourier transform. From (15) we find using IMS, 


$$
\begin{aligned}
E_{N-1, Z} \int \rho(x) \theta(x) d x \geqq & E_{N, Z} \int \rho(x) \theta(x) d x \\
\geqq & \sum_{i=1}^{N}\left\{\left\langle\psi\left|\sqrt{\theta\left(x_{i}\right)} H_{N-1, Z} \sqrt{\theta\left(x_{i}\right)}\right| \psi\right\rangle-\left\langle\psi\left|\left(\nabla \sqrt{\theta\left(x_{i}\right)}\right)^{2}\right| \psi\right\rangle\right. \\
& \left.+\left\langle\psi\left|-\theta\left(x_{i}\right) \frac{Z}{\left|x_{i}\right|}+\sum_{j, j \neq i} \frac{\theta\left(x_{i}\right)}{\left|x_{i}-x_{j}\right|}\right| \psi\right\rangle\right\} \\
\geqq & E_{N-1, Z} \int \rho(x) \theta(x) d x+N \int|\psi|^{2} K_{N-1} \theta(x) d x d x_{2} \cdots d x_{N} \\
& +C_{0} Z^{7 / 3-b} \int \rho(x) \theta(x) d x-c N R^{-2}-R^{-1} Z \int \rho(x) \theta(x) d x .
\end{aligned}
$$

Thus

$$
\begin{aligned}
& N \int|\psi|^{2}\left\|\nabla \chi_{x}\right\|_{L^{2}}^{2} K_{N-1} \theta(x) d x d x_{2} \cdots d x_{N} \\
& \quad \leqq C \sup _{x}\left\|\nabla \chi_{x}\right\|_{L^{2}}^{2}\left(\left(Z^{(7 / 3-b)}+R^{-1} Z\right) \int \rho \theta d x+Z R^{-2}\right) .
\end{aligned}
$$

Putting together (18),(19) and (20) gives (16).

A simplification of the above proof gives

Lemma 6. With the notation of Sect. 2,

$$
\left|\int\left(\rho(x)-\rho_{\mathrm{TF}}(x)\right) \lambda_{R}(x) d x\right| \leqq C\left(R^{1 / 2} Z^{(7 / 6-b / 2)}+R^{-1} Z^{1 / 3}\right) .
$$

\section{Estimates on $Q, v$ and $K$}

Estimate on $Q$. Using that $\rho_{\mathrm{TF}}(x)=Z^{2} \rho_{\mathrm{TF}}^{(Z=1)}\left(Z^{1 / 3} x\right)$, and

$$
|x| \geqq 1 \Rightarrow C_{-}|x|^{-6} \leqq \rho_{\mathrm{TF}}^{(1)}(x) \leqq C_{+}|x|^{-6},
$$

gives

$$
|x| \geqq Z^{-1 / 3} \Rightarrow C_{-}|x|^{-6} \leqq \rho_{\mathrm{TF}}(x) \leqq C_{+}|x|^{-6} .
$$

Furthermore $\int \rho_{\mathrm{TF}} d x=Z$. Thus

$$
\begin{aligned}
Q(R) & =N-\int \rho(x) \lambda_{R}(x) d x \\
& =N-\int \rho_{\mathrm{TF}}(x) \lambda_{R}(x) d x-\int\left(\rho(x)-\rho_{\mathrm{TF}}(x)\right) \lambda_{R}(x) d x \\
& =N-Z+\int \rho_{\mathrm{TF}}(x) \theta_{R}(x) d x-\int\left(\rho(x)-\rho_{\mathrm{TF}}(x)\right) \lambda_{R}(x) d x .
\end{aligned}
$$

Choose

$$
R=\gamma Z^{-1 / 3+b / 7}
$$

From

$$
C_{1} R^{-3} \leqq \int \rho_{\mathrm{TF}}(x) \theta_{R}(x) d x \leqq C_{2} R^{-3},
$$

and from Lemma 6 we then find

$$
\left(C_{1} \gamma^{-3}-C \gamma^{1 / 2}\right) Z^{(1-3 b / 7)} \leqq Q(R)-(N-Z) \leqq\left(C_{2} \gamma^{-3}+C \gamma^{1 / 2}\right) Z^{(1-3 b / 7)} .
$$

Choosing $\gamma$ appropriately $\left(\gamma \leqq(1 / 2)\left(C_{1} / C\right)^{2 / 7}\right.$ we have proved

Lemma 7. With $\alpha=3 b / 7$ and for $R=\gamma Z^{(1 / 3)(1-\alpha)}$ with $\gamma$ sufficiently small,

$$
0<c Z^{1-\alpha} \leqq Q(R)-(N-Z) \leqq C Z^{1-\alpha} \text {. }
$$


From the lower bound in (25) we get the result in Theorem 3 with $\alpha=3 b / 7$.

\section{Estimate on $v$.}

Lemma 8. For $\alpha$ and $R$ as in Lemma $\gamma$,

$$
v(R) \leqq C Z^{1-\alpha} .
$$

Proof. In (16) we choose $\theta(x)=\theta_{R}(x)$ and

$$
\chi(x, y)=\frac{|x|}{|x-y|} \lambda_{R(1-2 \delta)}(y) .
$$

For $(x, y) \in \operatorname{supp} \chi$ and $x \in \operatorname{supp} \theta_{R}$ we have $|x-y|>\delta R$. It is then easy to see that for $x \in \operatorname{supp} \theta_{R}$ we have

$$
\left\|\nabla_{y} \chi_{x}\right\|_{L^{2}\left(\mathbf{R}^{3}\right)} \leqq c R^{1 / 2} \text { and }\left\|\nabla \chi_{x}\right\|_{L^{\infty}} \leqq c R^{-1} .
$$

Taking this into account and remembering that $R=\gamma Z^{-(1 / 3)(1-\alpha)}$, we obtain from (16),

$$
\begin{aligned}
& \left|\int\left[\rho^{(2)}(x, y)-\rho_{\mathrm{TF}}(y) \rho(x)\right] \theta_{R}(x) \frac{|x|}{|x-y|} \lambda_{R(1-2 \delta)}(y) d x d y\right| \\
& \quad \leqq C_{\delta}\left(Q(R) Z^{1-\alpha}+Q(R)^{1 / 2} Z^{(2 / 3-\alpha / 6)}+Q(R) Z^{(2 / 3-\alpha / 3)}\right) \\
& \quad \leqq C_{\delta}\left(Q(R) Z^{1-\alpha}+Q(R)^{1 / 2} Z^{(2 / 3-\alpha / 6)}\right),
\end{aligned}
$$

where we have used that $b \leqq 2 / 3$ implies $\alpha \leqq 2 / 7$. From $\lambda_{R} \geqq \lambda_{(1-2 \delta) R}$ and Lemma 7 we can now conclude

$$
v(R) \leqq Z-\frac{1}{Q(R)} \int \rho_{\mathrm{TF}}(y) \rho(x) \theta_{R}(x) \frac{|x|}{|x-y|} \lambda_{R(1-2 \delta)}(y) d x d y+C_{\delta} Z^{1-\alpha} .
$$

Since $|x-y|^{-1}$ is the harmonic potential, $\lambda_{R(1-2 \delta)}$ and $\rho_{\mathrm{TF}}$ are spherically symmetric and $\lambda_{R(1-2 \delta)}$ is supported disjointly from $\theta_{R}$ we obtain

Recalling (22) we get

$$
v(R) \leqq Z-\int \rho_{\mathrm{TF}}(y) \lambda_{R(1-2 \delta)}(y) d y+C_{\delta} Z^{1-\alpha} .
$$

$$
v(R) \leqq \int \rho_{\mathrm{TF}}(y) \theta_{R(1-2 \delta)}(y) d y+C Z^{1-\alpha} \leqq C Z^{1-\alpha} .
$$

\section{Estimate on $K$}

Lemma 9. For $R$ as in Lemma 7,

$$
K(R) \geqq C Q(R) \quad \text { with } \quad C>0 .
$$

Proof. This can be done without the use of Lemma 5. Indeed notice that the inequality $\left\langle F^{2}\right\rangle-\langle F\rangle^{2} \geqq 0$ used on $F=\sum_{i=1}^{N} f\left(x_{i}\right)$ implies

$$
\int \rho^{(2)}(x, y) f(x) f(y) d x d y \geqq\left(\int \rho(x) f(x) d x\right)^{2}-\int \rho(x) f(x)^{2} d x .
$$

Hence since $\theta_{R} \leqq 1$,

$$
K(R) \geqq \frac{1}{Q(R)}\left(Q(R)^{2}-Q(R)\right)
$$




\section{and the result follows from Lemma 7.}

Proof of Theorem 1. Inserting the bounds from Lemmas 7-9 into the inequality of Theorem 4 gives the result of Theorem 1 .

Acknowledgements. We are grateful to C. Fefferman and E. Lieb for helpful and stimulating discussions.

\section{References}

1. Cycon, H. L., Froese, R. G., Kirsch, W., Simon, B.: Schrödinger Operators. Berlin, Heidelberg, New York: Springer 1987

2. Fefferman, C. L., Seco, L.A.: Asymptotic neutrality of large ions. Commun. Math. Phys. 128, 109-130 (1990)

3. Fefferman, C. L., Seco, L. A.: An upper bound for the number of electrons in a large ion. Proc. Natl. Acad. Sci. USA 86, 3464-3465 (1989)

4. Webster Hughes: An Atomic Energy Lower Bound that Gives Scott's Correction. PhD thesis,

5. Latter, R.: Atomic energy levels for the Thomas-Fermi and Thomas-Fermi-Dirac potential. Phys. Rev. 99, 510-599 (1955)

6. Lieb, E. H.: A lower bound for Coulomb energies. Phys. Lett. 70A, 444-446 (1979)

7. Lieb, E. H.: Thomas-Fermi and related theories of atoms and molecules. Rev. Mod. Phys. 53, 603-604 (1981)

8. Lieb, E. H.: Bound on the maximum negative ionization of atoms and molecules. Phys. Rev. A 29, 3018-3028 (1984)

9. Lieb, E. H., Sigal, I. M., Simon, B. Thirring, W.: Asymptotic neutrality of large-Z ions. Commun. Math. Phys. 116, 635-644 (1988)

10. Lieb, E. H., Simon, B.: The Thomas-Fermi theory of atoms, molecules, and solids. Adv. Math. 23, 22-116 (1977)

11. Ruskai, M. B.: Absence of discrete spectrum in highly negative ions. Commun. Math. Phys. 82, 457-469 (1982)

12. Ruskai, M. B.: Absence of discrete spectrum in highly negative ions, II. Commun. Math. Phys. 85, 325-327 (1982)

13. Siedentop, H., Weikard, R.: On the leading energy correction of the statistical model of the atom: Interacting case. Commun. Math. Phys. 112, 471-490 (1987)

14. Siedentop, H., Weikard, R.: On the leading correction of the Thomas-Fermi model: Lower bound - with an appendix by Müller, A. M. K., Invent. Math. 97, 159-193 (1989)

15. Sigal, I. M.: In: Proceeding of the VI International Congress on Mathematical Physics, W. Berlin, 1981

16. Sigal, I. M.: Geometric methods in the quantum many-body problem. Nonexistence of very negative ions. Commun. Math. Phys. 85, 309-324 (1982)

17. Sigal, I. M.: How many electrons can a nucleus bind? Ann. Phys. 157, 307-320 (1984)

18. Solovej, J. P.: Universality in the Thomas-Fermi-von Weizsäcker Theory of Atoms and Molecules. $\mathrm{PhD}$ thesis, Princeton, Department of Mathematics, June 1989

19. Thirring, W. E.: A lower bound with the best possible constant for Coulomb hamiltonians. Commun. Math. Phys. 79, 1-7 (1981)

20. Zhislin, G.: Discussion of the spectrum of Schrödinger operator for system of many particles. Tr. Mosk. Mat. Obs. 9, 81-128 (1960)

Communicated by B. Simon

Received October 30, 1989; in revised form January 23, 1990

Note added in proof. It has recently been shown (Fefferman, C. L., Seco, L. A.: The Ground State Energy of a Large Atom (To appear) that we can take the parameter $b$ equal to the optimal value $\frac{2}{3}$. This allows us to take $\alpha=\frac{2}{7}$. 
